\title{
Distribution pattern of black fly (Diptera: Simuliidae) assemblages along an altitudinal gradient in Peninsular Malaysia
}

\author{
Zubaidah Ya'cob ${ }^{1 *}$, Hiroyuki Takaoka' ${ }^{1}$ Pairot Pramual ${ }^{2}$, Van Lun Low ${ }^{1}$ and Mohd Sofian-Azirun ${ }^{1 *}$
}

\begin{abstract}
Background: Preimaginal black flies (Diptera: Simuliidae) are important components of the stream ecosystem. However, there has been limited research undertaken on the vertical distribution of preimaginal black flies and their associated ecological factors. Stream conditions are generally variable along the altitudinal gradient. Therefore, we conducted an in-depth entomological survey to investigate the simuliid distribution pattern along an altitudinal gradient in Peninsular Malaysia.
\end{abstract}

Methods: A total of 432 collections were performed in this study (24 samplings at each of 18 fixed-streams at monthly intervals) from February 2012 to January 2014. Larvae and pupae attached on aquatic substrates such as grasses, leaves and stems, twigs, plant roots and rocks were collected by hand using fine forceps. Stream depth $(\mathrm{m})$, width $(\mathrm{m})$, velocity $(\mathrm{m} / \mathrm{s})$, water temperature $\left({ }^{\circ} \mathrm{C}\right)$, acidity $(\mathrm{pH})$, conductivity $(\mathrm{mS} / \mathrm{cm})$ and dissolved oxygen $(\mathrm{mg} / \mathrm{L})$ were measured at the time of each collection.

Results: A total of 35 black fly species were recorded in the present study. The most frequently collected species were Simulium tani (31.7\%) and S. whartoni (21.5\%), while the relatively common species were Simulium sp. (nr. feuerborni) (16.2\%), S. decuplum (15.5\%), S. angulistylum (14.8\%), S. bishopi (13.2\%) and S. izuae (11.8\%). Total estimated species richness ranged between 39.8 and 41.3 , which yielded more than $80 \%$ of sampling efficiency. Six simuliid species were distributed below $500 \mathrm{~m}$, whereas eight species were distributed above $1400 \mathrm{~m}$. Simulium sp. (nr. feuerborni) and S. asakoae were found from middle to high altitudes (711-1813 m). Simulium whartoni, S. brevipar and S. bishopi were distributed widely from low to high altitudes (159-1813 m). Regression analysis between species richness and PCs revealed that the species richness was significantly associated with wider, deeper and faster streams at low altitude, normal water temperature $\left(23-25^{\circ} \mathrm{C}\right)$, low conductivity, higher discharge, more canopy cover and riparian vegetation and with larger streambed particles $(F=20.8, d f=1,422, P<0.001)$. Forward logistic regression indicated four species were significantly related to the stream variables (S. whartoni, Simulium sp. (nr. feuerborni), S. tani and S. angulistylum). Canonical correspondence analysis indicated that the temperature, stream size and discharge were the most important factors contributing to the separation of the stream sites from different altitude and hence are the predictors for the distribution of black fly species assemblages.

Conclusions: This study has provided insight into the distribution pattern of preimaginal black fly assemblages along an altitudinal gradient in Peninsular Malaysia. This study could deepen our knowledge on the ecology and biology of the specialised taxa in response to environmental changes.

Keywords: Black fly, Simulium, Elevation, Habitat characteristics, Peninsular Malaysia

\footnotetext{
*Correspondence: xuehy_perdana@yahoo.com; sofian@um.edu.my 'Institute of Biological Sciences, Faculty of Science, University of Malaya, 50603 Kuala Lumpur, Malaysia

Full list of author information is available at the end of the article
} 


\section{Background}

Species community structure and distribution vary spatially in response to a broad range of environmental factors including altitude $[1,2]$. Towards high altitude, the main general changes observed involve stream size [3], stream depth [4], temperature, precipitation (i.e. snow and rain), partial pressure of atmospheric gases, atmospheric turbulence and wind speed, and radiation input, including short-wave ultraviolet radiation at different wavelengths $[1,5]$. Consequently, all these changes create a barrier to species and drive to community diversification [2]. Moreover, communities appear to have been gradually decreasing in taxa richness with increasing altitude [6, 7].

Black flies (Diptera: Simuliidae) are insects of medical and veterinary significance. They are vectors of human diseases, notably human onchocerciasis or river blindness, the second ranking cause of infectious blindness [8]. In addition, black flies are also the vectors of diseases transmitted among wild animals and livestock $[9,10]$. On the other hand, black fly larvae are dominant inhabitants of unpolluted streams and rivers over a wide range of altitudes [11]. They are postulated to have evolved in cool and mountainous environments [8]. The climate change during the glacial periods has driven the population expansion of simuliids along latitudinal and altitudinal gradients $[8,12,13]$. However, as our climate changes, the widespread alpine taxa in the cooler regions could be fragmented and isolated as specialised taxa or high-altitude specialists [8].

Black fly assemblage is a colony of different species occurring in similar ecological or habitat requirements. Defining this assemblage is of paramount importance in evaluating the comparative richness of populations, and the effect of isolation or fragmentation [14]. Numerous factors have been linked to black flies assemblage, these include competition [15], food availability [16], substrate type $[17,18]$, water current velocity $[19,20]$, water temperature $[21,22]$ and altitude $[23,24]$. To date, the ecological studies of black flies have been given more attention on spatial distribution in response to habitat disturbance $[25,26]$, seasonal variation $[21,23]$ and locality richness [21, 27-30] as well as the breeding habitat preference [31]. However, there has been limited research undertaken on the vertical distribution of preimaginal black flies and their associated ecological factors. The knowledge of distribution patterns related to altitude could contribute to the understanding of the geographical distribution of many species as well as their local diversity.

We hypothesized that stream conditions vary according to the altitude, thus black fly diversity and assemblages are expected to change along the altitudinal gradient. Certain preimaginal stages of black flies would have a broad range of vertical distribution (i.e. generalist species), while others might show a specific range of distribution (i.e. specialist species). The specialised taxa may limit their distribution to certain preferred microhabitat/niche conditions [25, 31, 32]. To test this hypothesis, we made our first attempt to investigate the distribution pattern of black flies and their associated environmental factors, along an altitudinal gradient in Peninsular Malaysia.

\section{Methods \\ Study sites}

A total of 18 stream points were selected as fixed sites for sampling. Streams were chosen according to their accessibility for collection, altitude and the presence of flow. These stream locations were divided into three categories: (1) low altitude (100-500 m), (2) middle altitude (501-1000 m), (3) high altitude (1001-1813 m). A total of six streams were assigned as low altitude: (E1-E6), five streams as middle altitude: (E7-E11) and seven streams as high altitude (E12-E18). Seven streams are located in the state of Pahang (Cameron Highland, 04 ${ }^{\circ}$ $28.738^{\prime} \mathrm{N}, 101^{\circ} 22.979^{\prime} \mathrm{E}$ and Lipis, $04^{\circ} 23.715^{\prime} \mathrm{N}, 101^{\circ}$ $\left.36.443^{\prime} \mathrm{E}\right)$ while 11 streams are located in the state of Perak (Tapah, $04^{\circ} 14.203^{\prime} \mathrm{N}, 101^{\circ} 18.354$ 'E and Simpang Pulai, $\left.04^{\circ} 34.956^{\prime} \mathrm{N}, 101^{\circ} 20.717^{\prime} \mathrm{E}\right)$. Location details for 18 fixed-stream sites and associated ecological characteristics are presented in Fig. 1 and Table 1.

\section{Black fly sampling and identification}

Overall, a total of 432 collections were performed in this study (24 at each 18 fixed-stream sites at monthly intervals) from February 2012 to January 2014. Our extensive repeated samplings were expected to reveal the most accurate species number at all surveyed streams. Thus, sampling bias due to seasonal fluctuations in physicochemical parameters was minimized in this study. Each stream was sampled from downstream to upstream (20 m), for approximately $1 \mathrm{~h}$, by two people. Larvae and pupae attached on aquatic substrates such as grasses, leaves and stems, twigs, plant roots and rocks were collected by hand using fine forceps. These sampling protocols could represent the species occurrence in a locality [21, 33]. Pupae attached on similar substrates were individually kept alive in vials until emergence. The adults, together with their pupal exuviae and cocoons were preserved in $80 \%$ ethanol for identification at the subgenus, species-group or species level. The methods of collection and identification followed those of Takaoka [34] and Adler et al. [10].

\section{Physicochemical measurements}

The following stream physicochemical parameters were measured at the time of each collection: stream 


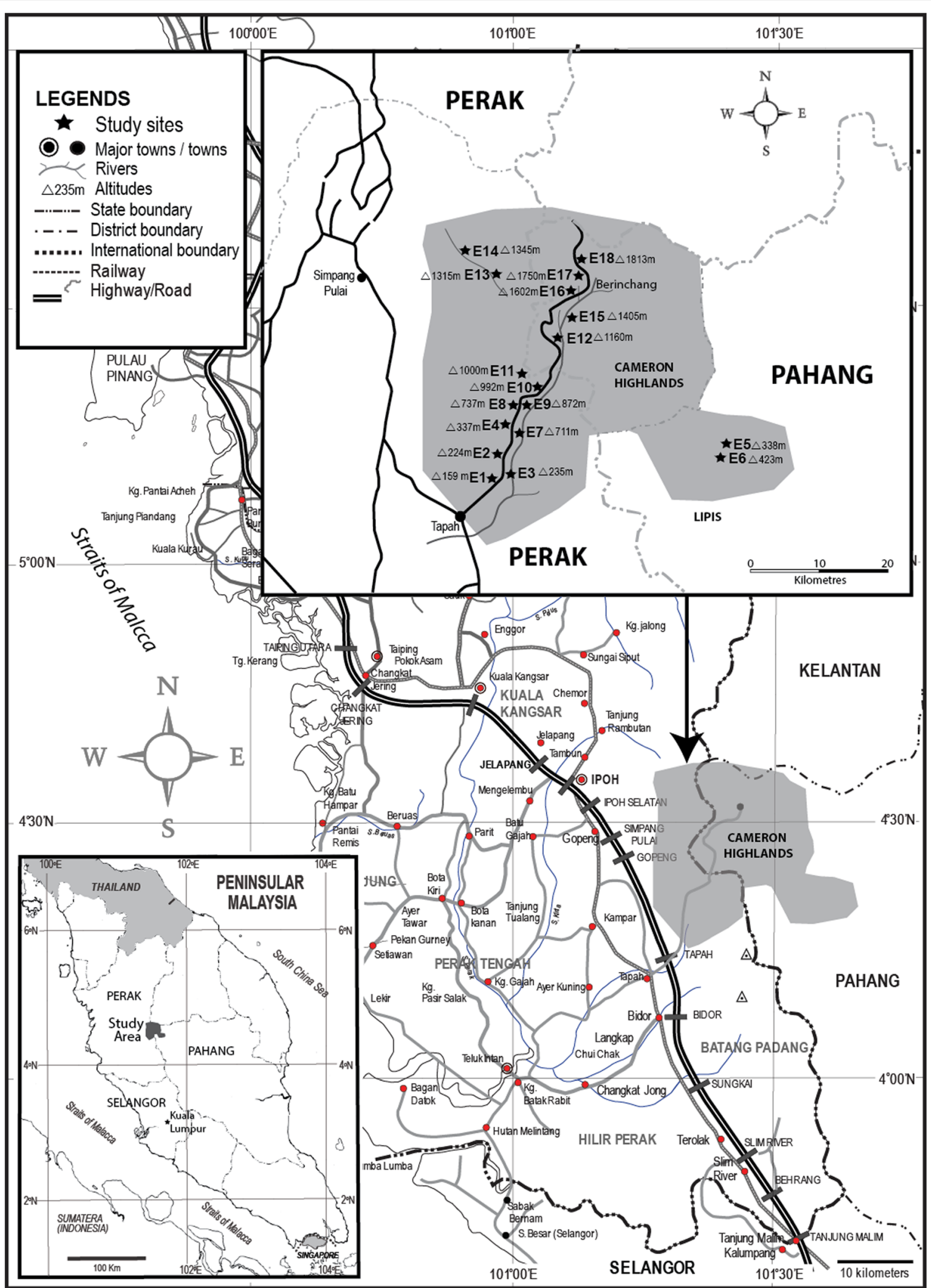

Fig. 1 Map showing 18 fixed-streams (E1 - E18) located in the states of Pahang and Perak in Peninsular Malaysia (top right), small map showing the Peninsular Malaysia (bottom left) 
Table 1 Locations of 18 fixed-streams and associated ecological characteristics

\begin{tabular}{|c|c|c|c|c|c|c|c|}
\hline Stream/altitude & Locality & Altitude (m) & Code & GPS & Canopy cover & Riparian vegetation & Streambed \\
\hline \multicolumn{8}{|l|}{ Low } \\
\hline E1 & Tapah & 159 & TPS1 & $04^{\circ} 14.203^{\prime} \mathrm{N}, 101^{\circ} 18.354^{\prime} \mathrm{E}$ & Partial & Forest & Rubble \\
\hline E2 & Tapah & 224 & TPS3 & $04^{\circ} 16.522^{\prime} \mathrm{N}, 101^{\circ} 18.996^{\prime} \mathrm{E}$ & Complete & Forest & Rubble \\
\hline E3 & Tapah & 235 & TPS2 & $04^{\circ} 16.316^{\prime} \mathrm{N}, 101^{\circ} 19.022^{\prime} \mathrm{E}$ & Open & Forest & Boulder \\
\hline E4 & Tapah & 337 & TPS4 & $04^{\circ} 18.420^{\prime} \mathrm{N}, 101^{\circ} 19.658^{\prime} \mathrm{E}$ & Complete & Forest & Bedrock \\
\hline E5 & Lipis & 388 & RBS18 & $04^{\circ} 23.715^{\prime} \mathrm{N}, 101^{\circ} 36.443^{\prime} \mathrm{E}$ & Open & Brush & Sand \\
\hline E6 & Lipis & 423 & RBS17 & $04^{\circ} 23.715^{\prime} \mathrm{N}, 101^{\circ} 36.443^{\prime} \mathrm{E}$ & Partial & Brush & Bedrock \\
\hline \multicolumn{8}{|l|}{ Middle } \\
\hline E7 & Cameron Highland & 711 & CHS5 & $04^{\circ} 22.220^{\prime} \mathrm{N}, 101^{\circ} 21.512^{\prime} \mathrm{E}$ & Partial & Forest & Bedrock \\
\hline E8 & Cameron Highland & 737 & CHS6 & $04^{\circ} 22.660^{\prime} \mathrm{N}, 101^{\circ} 21.902^{\prime} \mathrm{E}$ & Complete & Forest & Small stone \\
\hline E9 & Cameron Highland & 872 & CHS7 & $04^{\circ} 23.165^{\prime} \mathrm{N}, 101^{\circ} 22.334^{\prime} \mathrm{E}$ & Open & Forest & Boulder \\
\hline E10 & Cameron Highland & 992 & CHS9 & $04^{\circ} 24.274^{\prime} \mathrm{N}, 101^{\circ} 22.572^{\prime} \mathrm{E}$ & Partial & Forest & Bedrock \\
\hline E11 & Cameron Highland & 1000 & CHS8 & $04^{\circ} 24.112^{\prime} \mathrm{N}, 101^{\circ} 22.356^{\prime} \mathrm{E}$ & Complete & Forest & Sand \\
\hline \multicolumn{8}{|l|}{ High } \\
\hline E12 & Cameron Highland & 1160 & CHS10 & $04^{\circ} 26.723^{\prime} \mathrm{N}, 101^{\circ} 22.979^{\prime} \mathrm{E}$ & Partial & Forest & Sand \\
\hline E13 & Simpang Pulai & 1315 & SPS12 & $04^{\circ} 34.956^{\prime} \mathrm{N}, 101^{\circ} 20.717^{\prime} \mathrm{E}$ & Open & Brush & Bedrock \\
\hline E14 & Simpang Pulai & 1345 & SPS13 & $04^{\circ} 34.760^{\prime} \mathrm{N}, 101^{\circ} 20.507^{\prime} \mathrm{E}$ & Complete & Brush & Sand \\
\hline E15 & Cameron Highland & 1405 & $\mathrm{CHT11}$ & $04^{\circ} 28.738^{\prime} \mathrm{N}, 101^{\circ} 22.979^{\prime} \mathrm{E}$ & Partial & Forest & Rubble \\
\hline E16 & Cameron Highland & 1602 & CHS14 & $04^{\circ} 31.258^{\prime} \mathrm{N}, 101^{\circ} 24.247^{\prime} \mathrm{E}$ & Open & Brush & Boulder \\
\hline E17 & Cameron Highland & 1750 & CHS16 & $04^{\circ} 31.519^{\prime} \mathrm{N}, 101^{\circ} 23.365^{\prime} \mathrm{E}$ & Partial & Forest & Boulder \\
\hline E18 & Cameron Highland & 1813 & CHS15 & $04^{\circ} 31.461^{\prime} \mathrm{N}, 101^{\circ} 23.338^{\prime} \mathrm{E}$ & Complete & Brush & Small stone \\
\hline
\end{tabular}

depth $(\mathrm{m})$, width $(\mathrm{m})$, velocity $(\mathrm{m} / \mathrm{s})$ (one to three measurements along the collection path), water temperature $\left({ }^{\circ} \mathrm{C}\right)$, acidity $(\mathrm{pH})$, conductivity $(\mathrm{mS} / \mathrm{cm})$ and dissolved oxygen $(\mathrm{mg} / \mathrm{l})$. The values of $\mathrm{pH}$, temperature, conductivity and dissolved oxygen were taken using a portable multi probe parameter (Hanna HI 9828). Meter tape and steel ruler were used to measure stream width and depth, respectively, while a cork and a timer watch were used to measure stream velocity; the time taken for a cork to move one meter in distance. Velocity, depth and width measurements were used to estimate discharge [35]. The ecological and physicochemical measurement protocols including those for major streambed particles, riparian vegetation, and canopy cover followed those of McCreadie et al. [35]. For each fixed-stream site, the latitude and longitudinal coordinates were taken once and recorded using a hand held global positioning system (GPS) instrument (Garmin International Inc., Olathe, KS).

\section{Data analyses}

Frequency of occurrence (FO) was designated in percentages (Table 2), calculated by the total number of a species occurrence, divided by the total number of collections $(n=432)$ [30]. Stream occurrence $(\mathrm{SO})$ presented in percentages was calculated by the number of sites where a species was taken, divided by the total streams $(n=18)$. A rarefied species accumulation curve of individuals was created for all samples to determine if species in the site were adequately sampled [36]. The expected richness (First Order Jackknife and Chao estimates) was obtained to predict the possible number of species occurring in all fixed-stream sites. Species diversity estimations were calculated to determine the efficiency of the sampling by dividing the number of actual species collected by the number of estimated species [37]. To test the null hypothesis of random co-occurrence, we employed the null modeling software ECOSIM Version 7 [38] to create null models for co-occurrence, in which the C-score index [39] with fixed sums for row and column constraints was applied [40]. The presence or absence of a species was expressed on a binary scale $(0=$ absent, $1=$ present $)$, as in previous studies $[25,29,41]$. Cluster analysis based on Sorenson's coefficient was used to compare the percentage of site similarity in species composition for each site. Regression analysis was used to determine relationships between species distribution and stream variables. Because stream variables are inter-correlated, principal components analysis (PCA) was used to reduce the number of variables into groups of independent components. Principal components (PCs) with eigenvalues greater 
Table 2 Abundance of black flies, frequency of occurrence (FO) and stream occurrence (SO) from 24 collections at 18 fixed-streams in Peninsular Malaysia

\begin{tabular}{|c|c|c|c|}
\hline \multirow[t]{2}{*}{ Species } & \multirow{2}{*}{$\begin{array}{l}\text { Number of } \\
\text { specimens }\end{array}$} & $\%$ & \multirow{2}{*}{$\begin{array}{l}\% \\
\text { so }\end{array}$} \\
\hline & & $\mathrm{FO}$ & \\
\hline S. (Gomphostilbia) adleri Jitklang \& Kuvangkadilok, 2008 & 3 & 0.7 & 5.6 \\
\hline S. (Gomphostilbia) angulistylum Takaoka \& Davies, 1995 & 347 & 14.8 & 55.6 \\
\hline S. (Gomphostilbia) asakoae Takaoka \& Davies, 1995 & 837 & 7.6 & 55.6 \\
\hline S. (Gomphostilbia) brinchangense Takaoka et al., 2014 & 11 & 0.7 & 11.1 \\
\hline S. (Gomphostilbia) burtoni Takaoka \& Davies, 1995 & 19 & 3.0 & 16.7 \\
\hline S. (Gomphostilbia) cheongi Takaoka \& Davies, 1995 & 12 & 2.3 & 22.2 \\
\hline S. (Gomphostilbia) decuplum Takaoka \& Davies, 1995 & 176 & 15.5 & 61.1 \\
\hline S. (Gomphostilbia) duolongum Takaoka \& Davies, 1995 & 3 & 0.7 & 11.1 \\
\hline S. (Gomphostilbia) gombakense Takaoka \& Davies, 1995 & 117 & 10.0 & 50.0 \\
\hline S. (Gomphostilbia) izuae Takaoka et al., 2013 & 333 & 11.8 & 55.6 \\
\hline S. (Gomphostilbia) longitruncum Takaoka \& Davies, 1995 & 1 & 0.2 & 5.6 \\
\hline S. (Gomphostilbia) lurauense Takaoka et al., 2013 & 26 & 3.2 & 33.3 \\
\hline S. (Gomphostilbia) roslihashimi Takaoka \& Sofian-Azirun, 2011 & 112 & 9.3 & 55.6 \\
\hline S. (Gomphostilbia) sheilae Takaoka \& Davies, 1995 & 120 & 4.4 & 44.4 \\
\hline S. (Gomphostilbia) sofiani Takaoka \& Hashim, 2011 & 31 & 3.2 & 16.7 \\
\hline S. (Gomphostilbia) sp. (nr. parahiyangum) ${ }^{\mathrm{a}}$ & 54 & 6.5 & 38.9 \\
\hline S. (Gomphostilbia) tanahrataense Takaoka et al., 2014 & 1 & 0.2 & 5.6 \\
\hline S. (Gomphostilbia) trangense Jitklang et al., 2008 & 312 & 6.7 & 38.9 \\
\hline S. (Gomphostilbia) whartoni Takaoka \& Davies, 1995 & 709 & 21.5 & 77.8 \\
\hline S. (Nevermannia) aureohirtum Brunetti, 1911 & 103 & 4.9 & 27.8 \\
\hline S. (Nevermannia) caudisclerum Takaoka \& Davies, 1995 & 17 & 0.7 & 5.6 \\
\hline S. (Nevermannia) sp. (nr. feuerborni) ${ }^{a}$ & 688 & 16.2 & 44.4 \\
\hline S. (Nevermannia) kurtaki Takaoka \& Davies, 1995 & 1 & 0.2 & 5.6 \\
\hline S. (Simulium) bishopi Takaoka \& Davies, 1995 & 305 & 13.2 & 77.8 \\
\hline S. (Simulium) brevipar Takaoka \& Davies, 1995 & 122 & 10.6 & 77.8 \\
\hline S. (Simulium) digrammicum Edwards, 1928 & 1 & 0.2 & 5.6 \\
\hline S. (Simulium) grossifilum Takaoka \& Davies, 1995 & 106 & 8.3 & 50.0 \\
\hline S. (Simulium) hackeri Edwards, 1928 & 96 & 3.7 & 11.1 \\
\hline S. (Simulium) hirtinervis Edwards, 1928 & 63 & 3.0 & 33.3 \\
\hline S. (Simulium) jeffreyi Takaoka \& Davies, 1995 & 442 & 8.3 & 11.1 \\
\hline S. (Simulium) malayense Takaoka \& Davies, 1995 & 188 & 8.6 & 38.9 \\
\hline S. (Simulium) nobile De Meijere, 1907 & 42 & 1.6 & 5.6 \\
\hline S. (Simulium) sp. (nr. grisescens) ${ }^{a}$ & 7 & 0.2 & 5.6 \\
\hline S. (Simulium) tani Takaoka \& Davies, 1995 & 2669 & 31.7 & 66.7 \\
\hline S. (Simulium) yongi Takaoka \& Davies, 1997 & 11 & 1.9 & 16.7 \\
\hline
\end{tabular}

${ }^{a}$ Undetermined species, which probably are new species

than 1.0 were retained as variables. To interpret the PCs, Spearman's rank correlation test was used to detect the relationship between principal components and stream variables using a significance level of $P<0.001$. Forward logistic regression analysis was used to examine the relationships between spatial distribution and the PCs. Only species that occurred at more than $10 \%$ of total collections were considered in regression analyses [30] because those present at a lower frequency have resulted in the lack of statistical power (large number of zero values were observed) [21]. Linear regression was used to test the relationship between species richness (i.e. number of species in each sampling site) and the stream variables of the sampling sites (i.e. PC scores). All collections were 
subjected to PCA, and the PC scores were used for regression analysis. Canonical correspondence analysis (CCA) was used to investigate the relationship between environmental variables and species assemblages. CCA was analysed using the combined data set ( $n=432$ collections). The CCA was conducted using the program PC-ORD (version 5.14) [42]. Species Diversity and Richness (SDR) version 4 [43], the SPSS statistical package, version 16.0, Chicago, IL, were employed for diversity and statistical analyses respectively.

\section{Results}

\section{Black fly species composition}

Thirty-five species were collected from 24 samplings at each of 18 fixed-stream sites (Table 2). The most frequently collected species (FO) were $S$. $\operatorname{tani}(31.7 \%)$ and S. whartoni $(21.5 \%)$. Relatively common species were Simulium sp. (nr. feuerborni) (16.2 \%), S. decuplum (15.5\%), S. angulistylum (14.8\%), S. bishopi (13.2 \%) and $S$. izuae (11.8\%). Other species were collected at a frequency lower than $10 \%$ and considered as rare. In terms of total individuals collected, S. tani, S. asakoae, S. whartoni and Simulium sp. (nr. feuerborni) were the four most abundant species. Based on stream occurrence (SO), S. whartoni, S. bishopi and S. brevipar were the widest distributed species (14 streams or $77.8 \%$ each).

At the subgeneric level, Gomphostilbia was the most diverse subgenus found (19 species), followed by the Simulium (s. str.) (12 species) and the least was Nevermannia (four species). Of 18 species-groups in Malaysia, 17 were recorded in this study. The most abundant group was the $S$. asakoae species-group (six species), followed by the S. epistum species-group (four species). Other species groups were represented by one or two species.

Species richness and estimated richness are presented in Table 3. The maximum number of black fly species collected per total collections was 11 and the mean number was $3.2 \pm 0.1$ (SE). Total estimated species richness ranged between 39.8 and 41.3, which yielded more than $80 \%$ sampling efficiency, while the estimated species richness for each stream ranged between 4.1 and 24.0, with $60 \%$ sampling efficiency. Species reaching asymptote after approximately 54 samplings were performed, supporting the efficiency of the sampling method used in this study (Fig. 2).

Table 3 Actual and estimated species richness for 18 fixed-streams along an altitudinal gradient in Peninsular Malaysia. Numbers in parentheses indicate sampling efficiency ${ }^{a}$

\begin{tabular}{|c|c|c|c|c|}
\hline Altitudes & Actual species & Mean richness ( \pm SE) & Chao estimates & First Order Jackknife \\
\hline All & 35 & $3.15 \pm 2.00$ & $41.3 \pm 2.08(84.7)$ & $39.8 \pm 1.95(87.9)$ \\
\hline \multicolumn{5}{|c|}{ Low altitude } \\
\hline E1 & 13 & $2.52 \pm 0.35$ & $13.7 \pm 1.10(94.8)$ & $16.8 \pm 1.78(77.4)$ \\
\hline E2 & 15 & $3.43 \pm 0.31$ & $24.0 \pm 7.69(62.5)$ & $21.7 \pm 3.22(69.1)$ \\
\hline E3 & 15 & $3.50 \pm 0.28$ & $23.0 \pm 8.31(65.2)$ & $19.8 \pm 1.90(75.7)$ \\
\hline E4 & 8 & $3.21 \pm 0.41$ & $8.4 \pm 0.95(95.1)$ & $8.95 \pm 0.96(89.4)$ \\
\hline E5 & 12 & $4.30 \pm 0.52$ & $12.1 \pm 0.92(99.2)$ & $12.9 \pm 0.96(93.0)$ \\
\hline E6 & 14 & $4.64 \pm 0.48$ & $16.0 \pm 3.01(87.5)$ & $16.9 \pm 1.59(82.8)$ \\
\hline \multicolumn{5}{|c|}{ Middle altitude } \\
\hline E7 & 16 & $5.35 \pm 0.67$ & $18.0 \pm 3.01(88.8)$ & $17.9 \pm 1.33(89.4)$ \\
\hline E8 & 12 & $2.33 \pm 0.40$ & $16.1 \pm 11.5(74.5)$ & $17.7 \pm 2.07(67.8)$ \\
\hline E9 & 14 & $2.65 \pm 0.33$ & $20.3 \pm 5.89(68.9)$ & $18.8 \pm 1.95(74.5)$ \\
\hline E10 & 5 & $2.67 \pm 0.67$ & $7.0 \pm 3.01(71.4)$ & $7.8 \pm 2.87(64.1)$ \\
\hline E11 & 11 & $3.21 \pm 0.32$ & $13.6 \pm 7.78(80.9)$ & $13.9 \pm 1.59(79.1)$ \\
\hline \multicolumn{5}{|c|}{ High altitude } \\
\hline E12 & 11 & $3.44 \pm 0.35$ & $13.4 \pm 0.60(82.1)$ & $12.9 \pm 1.32(85.2)$ \\
\hline E13 & 16 & $3.34 \pm 0.43$ & $22.3 \pm 5.89(71.7)$ & $20.8 \pm 2.40(76.9)$ \\
\hline E14 & 13 & $3.36 \pm 0.50$ & $16.5 \pm 5.66(78.8)$ & $17.8 \pm 2.39(73.0)$ \\
\hline E15 & 13 & $2.83 \pm 0.28$ & $14.7 \pm 6.17(88.4)$ & $20.7 \pm 3.29(62.8)$ \\
\hline E16 & 4 & $1.69 \pm 0.15$ & $4.1 \pm 0.37(97.5)$ & $4.09 \pm 0.09(97.8)$ \\
\hline E17 & 7 & $1.29 \pm 0.18$ & $11.0 \pm 1.03(63.6)$ & $11.2 \pm 3.83(62.5)$ \\
\hline E18 & 10 & $1.76 \pm 0.42$ & $14.2 \pm 6.82(70.4)$ & $16.1 \pm 1.95(62.1)$ \\
\hline
\end{tabular}

${ }^{\mathrm{a} S a m p l i n g ~ e f f i c i e n c y ~ w a s ~ c a l c u l a t e d ~ b y ~ d i v i d i n g ~ t h e ~ n u m b e r ~ o f ~ a c t u a l ~ s p e c i e s ~ s a m p l e d ~ b y ~ t h e ~ n u m b e r ~ o f ~ e s t i m a t e d ~ s p e c i e s ~[36] ~}$ 


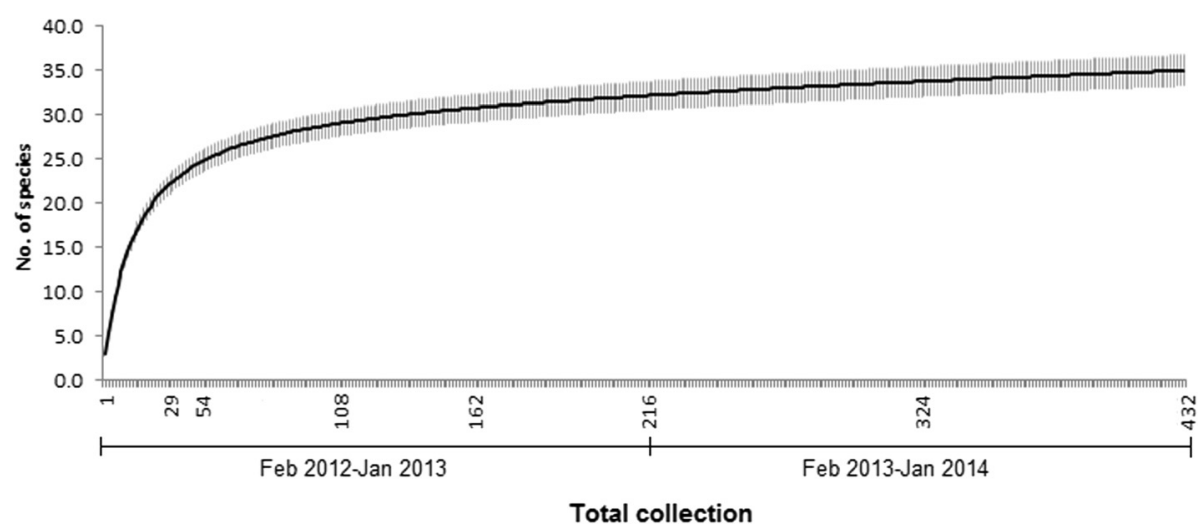

Fig. 2 Species accumulation curve with error bars for overall 432 collections at 18 fixed-streams along an altitudinal gradient in Peninsular Malaysia

\section{Species diversity and distribution patterns}

Diversity indices are presented in Fig. 3. Species diversity and richness increased with altitude and declined at $1600 \mathrm{~m}$ and above. Diversity value was highest at stream E8 (0.87) followed by streams E12 (0.81), E5 (0.79) and E13 (0.78). The values were lower at streams E17 (0.35), E18 (0.33), E16 (0.27) and E15 (0.25). In contrast, dominance index (D) was highest at $1400 \mathrm{~m}$ and above (E15-E18).

Frequency of occurrence for distribution of 35 black fly species is presented in Table 4. Five patterns of species distribution were observed: (1) six species distributed at low altitude (159-423 m), (2) three species distributed from low to middle altitudes (159-1000 m), (3) two species distributed from middle to high altitudes (711-1813 m) (4) eight species distributed at high altitudes (1405-1813 m) and (5) 16 species distributed from low to high altitudes (Table 4).
Of total collections $(n=432), 76.6 \%$ (or 331) showed co-existence of species. Simulated and observed values of the $\mathrm{C}$-score are presented in Fig. 4. The null model for co-occurrence indicates our observed index is above the simulated indices [observed index $=572.01$, mean of simulated indices $=558.83$, variance of simulated indices $=2.75, P($ observed $\leq$ expected $)=1.00, P($ observed $\geq$ ex pected $)=0.00$ ], therefore, the distributional patterns of species were not considered random.

Stream similarity based on species composition is presented in Fig. 5. Streams were clustered into two main groups with similarity value at $18 \%$ : $(1) \leq 1345 \mathrm{~m}$ (E1E14) and (2) $\geq 1405 \mathrm{~m}$ (E15-E18).

Means and coefficient of variations for eight measured physicochemical variables of all collections are presented in Table 5. PCA of all collections revealed five PCs, which have eigenvalues $>1.0$ accounted for $71.2 \%$ of total intersite variance of the physicochemical conditions (Table 6).

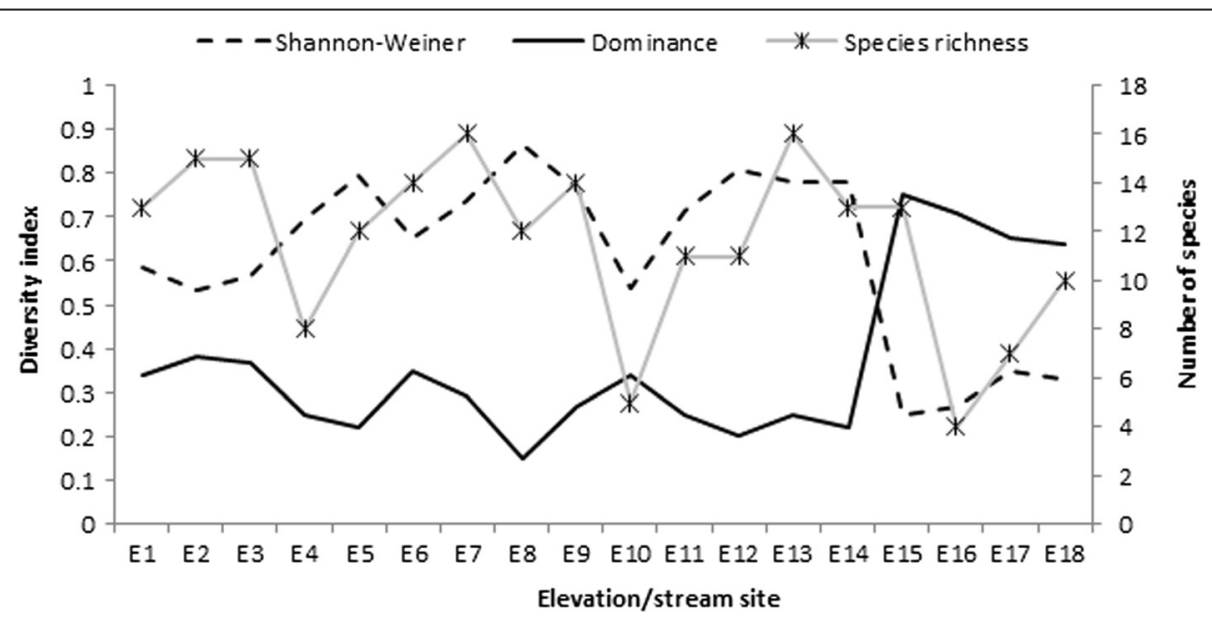

Fig. 3 Diversity indices for black fly species along an altitudinal gradient in Peninsular Malaysia 
Table 4 Frequency of occurrence per 24 collections for 35 black fly species along an altitudinal gradient in Peninsular Malaysia

\begin{tabular}{|c|c|c|c|c|c|c|c|c|c|c|c|c|c|c|c|c|c|c|}
\hline \multirow[t]{3}{*}{ Species } & \multicolumn{18}{|c|}{ Site } \\
\hline & \multicolumn{6}{|c|}{ Low } & \multicolumn{5}{|c|}{ Middle } & \multicolumn{7}{|c|}{ High } \\
\hline & E1 & E2 & E3 & E4 & E5 & E6 & E7 & E8 & E9 & E10 & E11 & E12 & E13 & E14 & E15 & E16 & E17 & E18 \\
\hline S. sp. (nr. grisescens) & & 1 & & & & & & & & & & & & & & & & \\
\hline S. duolongum & 2 & 1 & & & & & & & & & & & & & & & & \\
\hline S. nobile & & & 7 & & & & & & & & & & & & & & & \\
\hline S. cheongi & 2 & 1 & 3 & & 5 & & & & & & & & & & & & & \\
\hline S. adleri & & & & & & 3 & & & & & & & & & & & & \\
\hline S. jeffreyi & & 16 & 20 & & & & & & & & & & & & & & & \\
\hline S. lurauense & & 2 & 2 & & 3 & 1 & 5 & 1 & & & & & & & & & & \\
\hline S. yongi & & & & 3 & & & 2 & & 3 & & & & & & & & & \\
\hline S. sheilae & 7 & 2 & 1 & 1 & 3 & 2 & 1 & & & & 2 & & & & & & & \\
\hline S. angulistylum & 16 & 2 & 4 & 5 & 13 & 18 & 2 & & & 1 & 2 & 1 & & & & & & \\
\hline S. sp. (nr. parahiyangum) & 1 & 15 & 6 & & 3 & 1 & & & 1 & & & & 1 & & & & & \\
\hline S. roslihashimi & 6 & & 1 & & 1 & 5 & 7 & 4 & 1 & & 12 & 1 & & 2 & & & & \\
\hline S. trangense & 15 & 4 & 4 & & & 3 & & 1 & 1 & & & & & 1 & & & & \\
\hline S. grossifilum & 1 & 1 & 2 & 8 & & & 16 & 1 & 6 & & & & 1 & 1 & & & & \\
\hline S. malayense & & 1 & & & & 5 & 18 & 6 & 5 & & & & 1 & 1 & & & & \\
\hline S. izuae & 1 & & & & & 1 & 7 & 8 & 2 & & 13 & 10 & 2 & 6 & 1 & & & \\
\hline S. burtoni & & 5 & 7 & & & & & & & & & & & & 1 & & & \\
\hline S. hirtinervis & & 1 & 1 & & & & 6 & & & & & & 1 & & 1 & 3 & & \\
\hline S. decuplum & 2 & & 2 & & 16 & 14 & 10 & & 3 & & & 9 & 6 & 2 & 1 & & 1 & \\
\hline S. gombakense & & & & & 12 & 11 & 2 & 3 & 1 & 2 & 6 & 6 & & & & & 1 & \\
\hline S. brevipar & 2 & & & 2 & 2 & 8 & 3 & 2 & 5 & 3 & 2 & 5 & 3 & 7 & & & 1 & 1 \\
\hline S. bishopi & 1 & & & 9 & 9 & 4 & 11 & 1 & 1 & 1 & 1 & 4 & 8 & 5 & 1 & & & 1 \\
\hline S. aureohirtum & 2 & & & & & & & & & & & & 4 & & 4 & 10 & & 1 \\
\hline S. $\tan i$ & & 22 & 21 & 14 & 3 & & 21 & 1 & 11 & & 1 & 4 & 14 & 5 & 20 & & & 1 \\
\hline S. whartoni & & 1 & 1 & 3 & 14 & 19 & 6 & 3 & 2 & 1 & 15 & 9 & 5 & 11 & 1 & & 1 & 1 \\
\hline S. asakoae & & & & & & & 1 & 1 & 1 & & 1 & 3 & 2 & & 1 & 21 & 2 & 2 \\
\hline S. sp. (nr. feuerborni) & & & & & & & & & & & 2 & 6 & 4 & 4 & 19 & 6 & 14 & 15 \\
\hline S. digrammicum & & & & & & & & & & & & & 1 & & & & & \\
\hline S. longitruncum & & & & & & & & & & & & & & 1 & & & & \\
\hline S. hackeri & & & & & & & & & & & & & 9 & & 7 & & & \\
\hline S. sofiani & & & & & & & & & & & & & 4 & 1 & 9 & & & \\
\hline S. tanahrataense & & & & & & & & & & & & & & & 1 & & & \\
\hline S. brinchangense & & & & & & & & & & & & & & & & & 2 & 1 \\
\hline S. kurtaki & & & & & & & & & & & & & & & & & & 1 \\
\hline S. caudisclerum & & & & & & & & & & & & & & & & & & 3 \\
\hline
\end{tabular}

Spearman's rank correlations revealed that sites with higher PC-1 (which explained $24.8 \%$ of the total variance) were at low altitude, normal water temperature $\left(23-25^{\circ} \mathrm{C}\right)$, wider, deeper, faster, low conductivity, higher discharge, more canopy cover and riparian vegetation and with larger streambed particles. Sites with higher PC-2 (which explained $15.1 \%$ of the total variance) scores were at high altitude, cooler stream, wider, faster, higher dissolved oxygen with high discharge, less canopy cover and riparian vegetation. PC-3 accounted for $13.8 \%$ of the total variance. Sites with higher PC-3 scores were at high altitude, cooler stream, smaller, slower, low conductivity and $\mathrm{pH}$ with low discharge and smaller streambed particles. PC-4 explained $9.3 \%$ of the total intersite variance. Sites with higher PC-4 


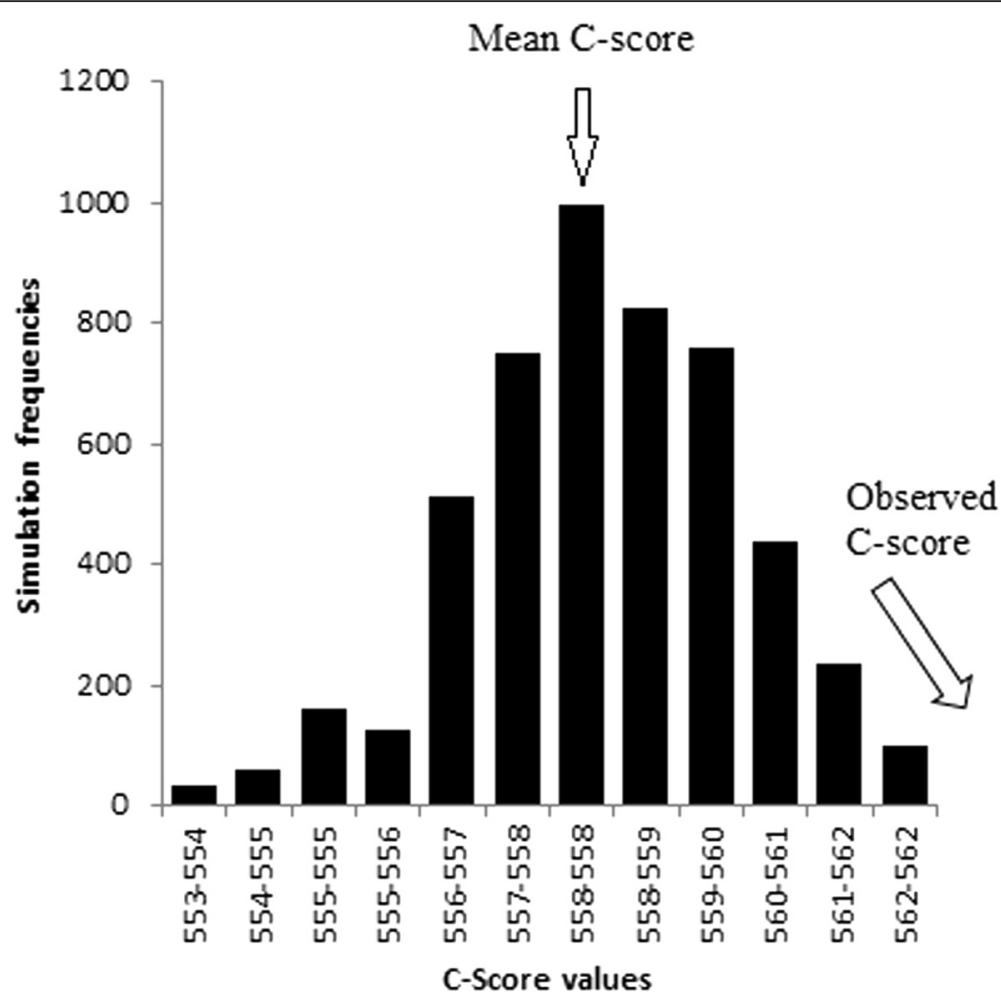

Fig. 4 Simulated and observed values of the C-score for the co-occurrence of the simuliid species collected at 18 fixed-streams along an altitudinal gradient in Peninsular Malaysia

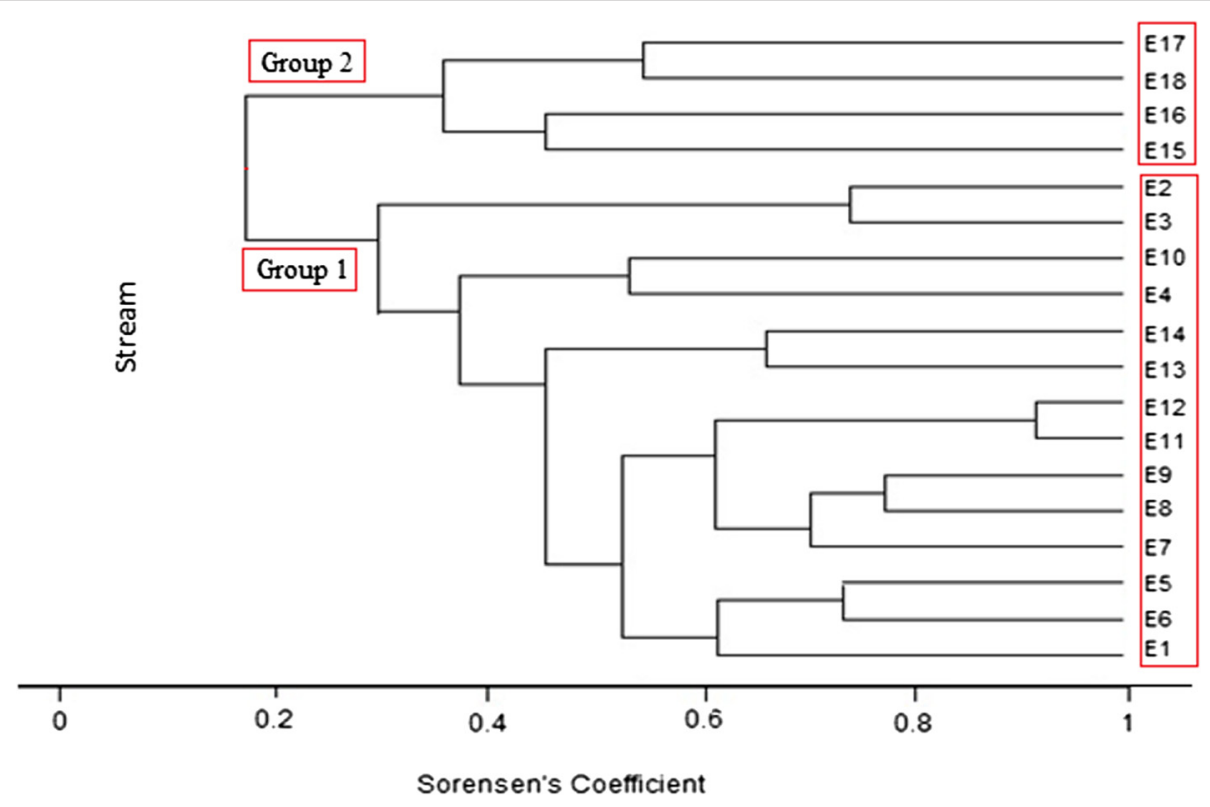

Fig. 5 Cluster analysis based on Sorenson's coefficient for site similarity along an altitudinal gradient in Peninsular Malaysia 
Table 5 Physicochemical characteristics of all study sites presented as mean and coefficient of variation (CV)

\begin{tabular}{|c|c|c|c|c|c|c|c|c|c|c|}
\hline Altitude/Site & & Parameter & $\begin{array}{l}\text { Temperature } \\
\left({ }^{\circ} \mathrm{C}\right)\end{array}$ & Width (m) & Depth (m) & Velocity (m/s) & Conductivity (mS/cm) & Do (mg/l) & $\mathrm{pH}$ & Discharge $\left(\mathrm{m}^{3} / \mathrm{s}\right)$ \\
\hline \multirow[t]{12}{*}{ Low altitudes } & E1 & mean & 25.03 & 0.89 & 0.14 & 0.42 & 0.15 & 4.84 & 6.51 & 0.07 \\
\hline & & CV & 4.89 & 106.67 & 47.46 & 14.73 & 19.77 & 76.62 & 10.8 & 157.53 \\
\hline & E2 & mean & 24.13 & 5.38 & 0.18 & 0.47 & 0.20 & 5.54 & 6.79 & 0.47 \\
\hline & & CV & 3.12 & 16.83 & 31.99 & 27.11 & 18.72 & 68.29 & 8.88 & 55.1 \\
\hline & E3 & mean & 24.26 & 5.7 & 0.16 & 0.44 & 0.18 & 5.67 & 6.93 & 0.41 \\
\hline & & CV & 11.24 & 28.31 & 42.37 & 21.51 & 23.96 & 71.67 & 12.39 & 73.48 \\
\hline & E4 & mean & 22.55 & 3.15 & 0.19 & 0.65 & 0.14 & 5.37 & 6.99 & 0.36 \\
\hline & & $C V$ & 5.09 & 49.38 & 39.05 & 32.43 & 11.39 & 53.42 & 11.00 & 61.65 \\
\hline & E5 & mean & 23.92 & 0.61 & 0.12 & 0.59 & 0.44 & 5.94 & 6.85 & 0.04 \\
\hline & & $C V$ & 4.12 & 30.35 & 34.65 & 21.93 & 16.23 & 33.45 & 9.65 & 52.67 \\
\hline & E6 & mean & 23.39 & 0.88 & 0.1 & 0.52 & 0.45 & 5.84 & 7.21 & 0.05 \\
\hline & & CV & 5.33 & 30.8 & 59.13 & 30.36 & 21.99 & 30.43 & 8.08 & 78.22 \\
\hline \multirow[t]{10}{*}{ Middle altitudes } & E7 & mean & 21.68 & 1.64 & 0.22 & 0.53 & 0.34 & 5.54 & 7.31 & 0.23 \\
\hline & & CV & 5.91 & 38.3 & 66.78 & 17.85 & 18.73 & 39.59 & 6.62 & 130.2 \\
\hline & E8 & mean & 21.55 & 0.52 & 0.05 & 0.34 & 0.60 & 5.48 & 7.34 & 0.01 \\
\hline & & $C V$ & 4.09 & 38.99 & 29.14 & 23.56 & 13.75 & 38.92 & 7.12 & 53.62 \\
\hline & E9 & mean & 20.21 & 1.81 & 0.14 & 0.52 & 0.33 & 6.35 & 7.24 & 0.15 \\
\hline & & CV & 5.15 & 50.59 & 41.25 & 31.59 & 14.7 & 35.53 & 7.80 & 84.73 \\
\hline & E10 & mean & 20.42 & 0.93 & 0.14 & 0.38 & 0.25 & 7.52 & 7.52 & 0.07 \\
\hline & & CV & 2.98 & 61.12 & 44.8 & 6.00 & 50.00 & 7.63 & 5.13 & 73.4 \\
\hline & E11 & mean & 19.36 & 0.37 & 0.09 & 0.35 & 0.43 & 5.18 & 7.06 & 0.01 \\
\hline & & $C V$ & 3.97 & 57.45 & 88.63 & 23.1 & 25.51 & 43.15 & 8.04 & 116.13 \\
\hline \multirow[t]{14}{*}{ High altitudes } & E12 & mean & 17.71 & 0.56 & 0.09 & 0.34 & 0.17 & 3.89 & 7.00 & 0.02 \\
\hline & & $C V$ & 5.69 & 38.44 & 41.64 & 24.44 & 49.45 & 59.67 & 8.67 & 75.36 \\
\hline & E13 & mean & 18.65 & 3.07 & 0.07 & 0.67 & 0.53 & 6.27 & 7.19 & 0.16 \\
\hline & & CV & 6.46 & 49.6 & 103.01 & 23.95 & 24.91 & 29.87 & 12.35 & 134.23 \\
\hline & E14 & mean & 18.34 & 0.73 & 0.05 & 0.39 & 0.23 & 6.49 & 6.72 & 0.01 \\
\hline & & CV & 4.13 & 15.05 & 34.01 & 42.18 & 18.2 & 18.95 & 11.36 & 54.31 \\
\hline & E15 & mean & 17.22 & 3.17 & 0.22 & 0.54 & 0.18 & 5.94 & 6.79 & 0.37 \\
\hline & & $C V$ & 4.93 & 20.22 & 24.45 & 25.36 & 33.85 & 34.93 & 9.76 & 50.13 \\
\hline & E16 & mean & 17.9 & 1.03 & 0.15 & 0.42 & 0.33 & 5.66 & 6.65 & 0.09 \\
\hline & & $C V$ & 6.72 & 127.76 & 43.18 & 28.77 & 51.06 & 32.39 & 10.53 & 194.76 \\
\hline & E17 & mean & 17.99 & 0.62 & 0.14 & 0.33 & 0.18 & 5.23 & 6.56 & 0.02 \\
\hline & & $C V$ & 6.57 & 37.14 & 174.22 & 36.32 & 33.86 & 42.58 & 9.21 & 117.08 \\
\hline & E18 & mean & 17.39 & 1.35 & 0.06 & 0.37 & 0.71 & 4.31 & 6.49 & 0.03 \\
\hline & & CV & 8.09 & 129.59 & 38.68 & 69.16 & 93.68 & 61.94 & 8.78 & 154.23 \\
\hline
\end{tabular}

scores were at high altitude, cooler stream, high dissolved oxygen and $\mathrm{pH}$ with more canopy covers and larger streambed particles. PC-5 accounted for $8.4 \%$ of the total variance. Streams with higher PC-5 scores were faster, high conductivity, low oxygen, more stream canopy covers and larger streambed particles. Regression analysis revealed that species richness was significantly associated with PC-1 ( $F=$ 20.8, $d f=1,422, P<0.001)$.
Regression analysis for four black fly species is presented in Table 7. Forward logistic regression analyses were conducted for eight species, which were found in more than $10 \%$ (FO) of total collections. All regression models of species distribution except S. decuplum, S. izuae, S. brevipar and S. bishopi, were significant at $P<0.001$ with correct classification varying from 73.4 to $85.3 \%$. Simulium whartoni was positively associated with PC-1 and PC-3. 
Table 6 Principal component analysis and Spearman's rank correlation coefficients between stream variables and principal components for all collections $(n=432)$

\begin{tabular}{|c|c|c|c|c|c|c|c|c|}
\hline \multirow[t]{2}{*}{ Variable } & \multicolumn{3}{|c|}{ Stream sites } & \multicolumn{5}{|c|}{ Principal components } \\
\hline & Min & Max & Mean \pm SE & $P C-1$ & $P C-2$ & $P C-3$ & $P C-4$ & $P C-5$ \\
\hline Altitude (m) & 159.00 & 1813.00 & $891.40 \pm 30.1$ & $-0.751^{* *}$ & $0.268^{* *}$ & $0.522^{* *}$ & $0.224^{* *}$ & 0.049 \\
\hline Temperature $\left({ }^{\circ} \mathrm{C}\right)$ & 14.40 & 28.40 & $25.30 \pm 0.17$ & $0.636^{* *}$ & $-0.230^{* *}$ & $-0.622^{* *}$ & $-0.285^{* *}$ & -0.059 \\
\hline Width (m) & 0.12 & 7.90 & $2.95 \pm 0.11$ & $0.692^{* *}$ & $0.322^{* *}$ & $-0.132^{* *}$ & 0.044 & 0.037 \\
\hline Depth (m) & 0.02 & 0.85 & $0.54 \pm 0.005$ & $0.616^{* *}$ & 0.171 & 0.34 & 0.034 & 0.124 \\
\hline Velocity $(\mathrm{m} / \mathrm{s})$ & 0.16 & 1.03 & $0.42 \pm 0.009$ & $0.416^{* *}$ & $0.544^{* *}$ & $-0.217^{* *}$ & 0.115 & $0.360^{* *}$ \\
\hline Conductivity (mS/cm) & 0.01 & 0.15 & $3.48 \pm 0.001$ & $-0.297^{* *}$ & 0.162 & $-0.327^{* *}$ & 0.001 & $0.328^{* *}$ \\
\hline Dissolved oxygen (mg/l) & 1.27 & 17.20 & $15.77 \pm 0.14$ & 0.148 & $0.336^{* *}$ & -0.051 & $0.363^{* *}$ & $-0.576^{* *}$ \\
\hline $\mathrm{pH}$ & 4.32 & 8.53 & $5.20 \pm 0.04$ & 0.036 & -0.080 & $-0.337^{* *}$ & $0.739^{* *}$ & -0.155 \\
\hline Discharge $\left(\mathrm{m}^{3} / \mathrm{s}\right)$ & 0.002 & 1.56 & $2.23 \pm 0.01$ & $0.786^{* *}$ & $0.376^{* *}$ & $-0.208^{* *}$ & 0.049 & 0.137 \\
\hline Canopy cover & open & complete & $2^{\mathrm{a}}$ & $0.213^{* *}$ & $-0.718^{* *}$ & 0.175 & $0.315^{* *}$ & $0.306^{* *}$ \\
\hline Riparian & open & forest & $2^{a}$ & $0.400^{* *}$ & $-0.763^{* *}$ & 0.174 & 0.122 & -0.015 \\
\hline Streambed & sand & bedrock & $3^{\mathrm{a}}$ & $0.449^{* *}$ & 0.176 & $-0.503^{* *}$ & $0.274^{* *}$ & $0.366^{* *}$ \\
\hline \multicolumn{9}{|c|}{$\%$ Variance explained in PCA } \\
\hline Proportion & & & & 24.8 & 15.1 & 13.8 & 9.3 & 8.4 \\
\hline Cumulative & & & & 24.8 & 39.7 & 53.6 & 62.8 & 71.2 \\
\hline
\end{tabular}

** $P<0.001$ cover. ( 1 = open, 2 = partial and 3 = complete). Rankings followed McCreadie et al. [34]

Simulium sp. (nr. feuerborni) was positively associated with PC-3, S. tani was positively associated with PC-1, PC-2 and PC-4. Simulium angulistylum was positively associated with PC-1 but negatively associated with PC-3 and PC-4. PC-5 was not related to any species.

An ordination diagram for 18 fixed-stream sites and species are presented in Figs. 6 and 7, respectively. CCA indicated that temperature, stream size and discharge were the most important factors in differentiating streams from different altitudes. Therefore, these factors are good predictors for black fly species assemblages. The relationship between species and stream variable conditions was high (>0.569) for the first three canonical axes, indicating that the variables used in this study were strongly related to black fly species assemblage. Temperature was the most important factor on the CCA axis 1. Species that associated with normal stream temperature were $S$. cheongi and S. trangense. The bottom left panel of the biplot is characterized by streams with wider and higher discharge. These sites were predominated by $S$. tani, S. nobile and S. jeffreyi. The upper right side of the biplot is composed of sites with lower discharge and smaller streams. Black fly species found predominantly at these sites were $S$. bishopi, $S$. izuae and $S$. longitruncum. The bottom right panel of the biplot is characterized by low water temperature, which is characteristic of high altitude streams. Black flies predominating at these sites were S. asakoae, S. caudisclerum and Simulium sp (nr. feuerborni) (Fig. 7).

\section{Discussion}

This in-depth survey on the vertical distribution of black flies was conducted for the first time in Peninsular Malaysia and yielded 35 species, representing $42.7 \%$ of total simuliids in Malaysia (82 species) [44]. Simulium digrammicum from Cameron Highland, an earlier described species and previously considered as locally extinct, was discovered in our study. These findings

Table 7 Regression analysis for the distribution of preimaginal black fly species at 18 fixed-streams along an altitudinal gradient in Peninsular Malaysia

\begin{tabular}{|c|c|c|c|c|c|c|c|c|}
\hline \multirow[t]{2}{*}{ Species } & \multicolumn{6}{|c|}{ Regression coefficient } & \multirow[t]{2}{*}{ P } & \multirow{2}{*}{$\begin{array}{l}\% \\
\text { Correc }\end{array}$} \\
\hline & K & $P C-1$ & $P C-2$ & $P C-3$ & $P C-4$ & $P C-5$ & & \\
\hline Simulium whartoni & -1.048 & 0.403 & - & 0.741 & - & - & $<0.001$ & 73.4 \\
\hline Simulium sp.(nr. feuerborni) & -1.449 & - & - & 1.214 & - & - & $<0.001$ & 78.1 \\
\hline Simulium tani & -0.461 & 1.837 & 0.590 & - & 0.393 & - & 0.001 & 79.3 \\
\hline Simulium angulistylum & -2.463 & 0.805 & - & -2.012 & -0.444 & - & $<0.001$ & 85.3 \\
\hline
\end{tabular}




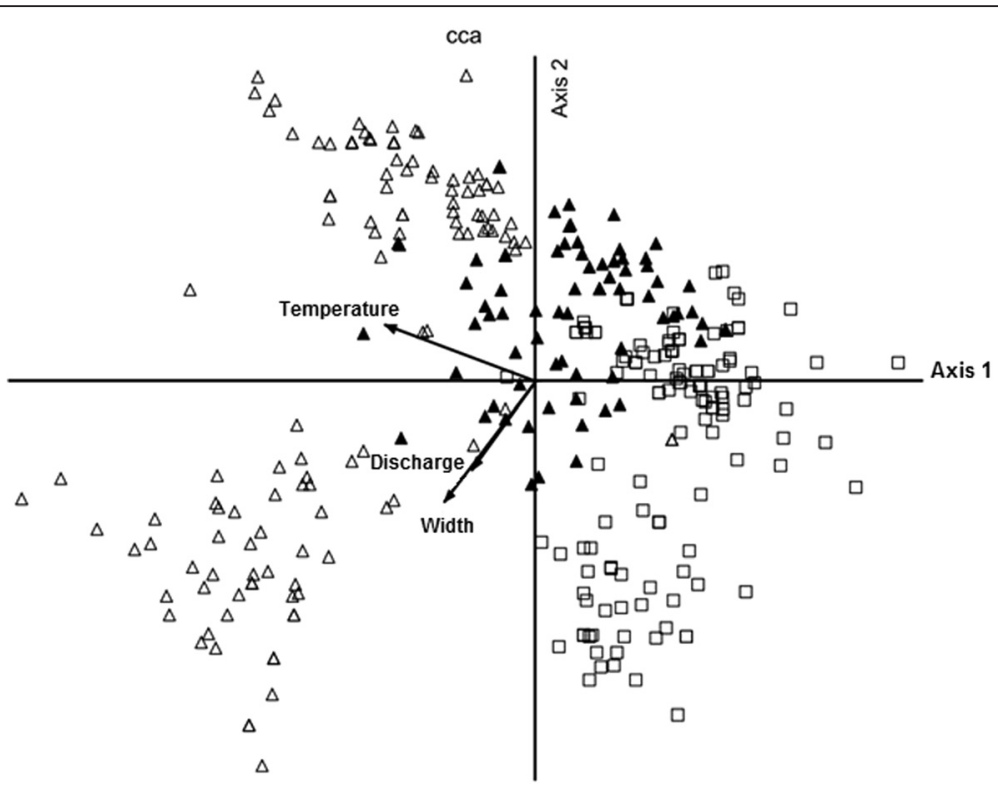

Fig. 6 Ordination diagram of the first two axes of canonical correspondence analysis (CCA) of 432 sampling collections (open triangles represent low-altitude sites; closed triangles represent middle-altitude sites; and open square represent high-altitude sites). Arrows denote environmental variables with strength of the environmental condition indicated by arrow length of closeness to the CCA axis

indicated that all sampled streams are the natural breeding habitats for black flies. As far as medico-veterinary importance of black flies is concerned, we collected $S$. (G.) asakoae, one of the well-known species that have been reported to be infected with filarial parasites in
Thailand [45]. The host biting habits and vectorial capacity of Malaysian black flies, however, remain unknown. Average number of species per total collections in this study was 3.2, slightly higher than previously reported $[21,25,30-32]$. Our study also revealed $74.3 \%$ of the

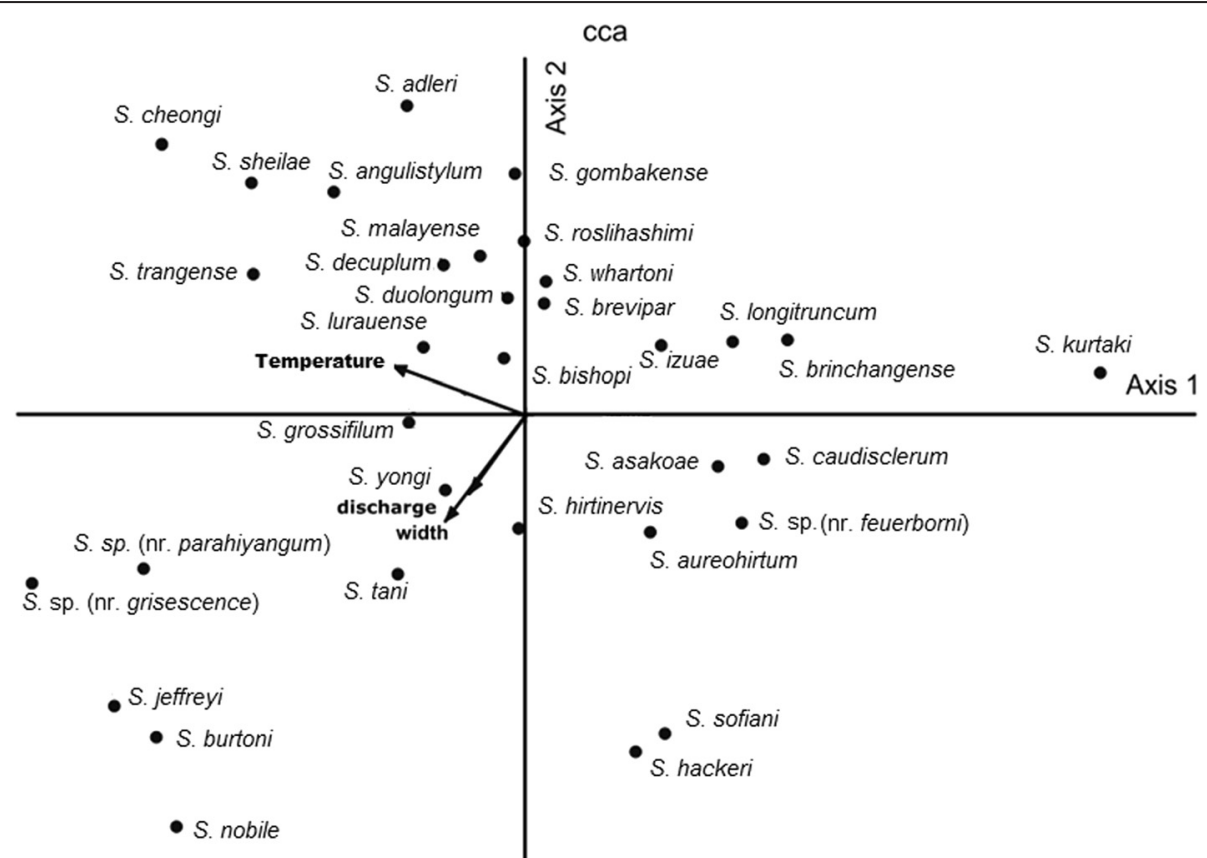

S. digrammicum
$\bullet \quad$ S. tanahrataense

Fig. 7 Ordination diagram of the first two axes of canonical correspondence analysis (CCA) of the 35 black fly species in Peninsular Malaysia 
sampled black flies as rare species $(\mathrm{FO}<10 \%)$. This pattern was consistent with our previous study in Peninsular Malaysia [31] and other geographical regions [26, 30, 46]. In contrast, $22.8 \%$ (eight species) of total species had higher frequency of occurrence (10.6 to $31.7 \%$ ), stream occurrence (44.4 to $77.8 \%$ ) and total specimens collected, confirming the previous observations where the species were relatively abundant, on average and also widely distributed $[47,48]$. The capability of these species to adapt over a broad range of stream physicochemical conditions has allowed them to occur almost in all places and become generalist. In contrast, rare species require a more specialised habitat, which consequently limits their distribution to certain streams conditions and defines them as specialist. This situation also corroborated with the prediction on taxa distribution [49] and neutral theory [50].

This study showed that the five principal components had eigenvalues $>1.0$ and accounted for $71.2 \%$ of total intersite variance of the stream condition variables. Streams at low altitude, with normal water temperature $\left(23-25{ }^{\circ} \mathrm{C}\right)$, wider, deeper and faster, low conductivity, higher discharge, more canopy covers and riparian vegetations and with larger stream-bed particles, accommodate more preimaginal black fly species. Some of these core factors were consistent with previous studies [21, 23, 30-32, 51, 52].

The first two CCA axes indicated the differentiation of low, middle and high altitude streams (Fig. 5). As expected, temperature, stream size and discharge are varied as altitude increases. The general conditions of low altitude streams were warmer, wider and higher discharge with average mean $23.8{ }^{\circ} \mathrm{C}, 2.9 \mathrm{~m}$, and $0.2 \mathrm{~m}^{3} / \mathrm{s}$ respectively. High altitude sites were cooler $\left(17.8{ }^{\circ} \mathrm{C}\right)$ than middle altitude sites $\left(20.6{ }^{\circ} \mathrm{C}\right)$. However, the average mean for stream size and discharge observed both at middle $\left(1.0 \mathrm{~m} ; 0.09 \mathrm{~m}^{3} / \mathrm{s}\right)$ and high $\left(1.5 \mathrm{~m} ; 0.1 \mathrm{~m}^{3} / \mathrm{s}\right)$ altitudes were less different. In fact, stream sites at these altitudes are smaller and slower compared to low altitude sites. Our results showed gradual decrease of water temperature values as altitude increased. Dudgeon [53] suggested low water temperature as the most characteristic feature of high altitude streams. This finding is consistent with previous studies in other geographical regions [3, 4]. Besides, Tomanova et al. [4] reported stream depth as another factor that negatively related to altitude. Regarding difference in altitudes, Srisuka et al. [23] indicated that certain Thai simuliids occurred exclusively in a single zone while others were found in almost all gradients. This result was consistent with a previous study on other aquatic macroinvertebrates [3]. Our study corroborates this trend, species such as Simulium sp. (nr. feuerborni) of the subgenus Nevermannia and S. asakoae of the subgenus Gomphostilbia were found to be restricted to middle and high altitudes. The Thai S. asakoae, however, was distributed from low to high altitudes (500-2100 m) with predominance at low altitude [23]. Based on these distinct ecological conditions between Thai populations and Malaysian populations, coupled with the previous genetic evidence [54], we suggest the presence of cryptic species in S. asakoae. Three species in each of the subgenus Gomphostilbia (S. duolongum, S. cheongi and S. adleri) and the subgenus Simulium (S. nobile, S. jeffreyi and Simulium sp. nr. grisescens) were distributed at low altitude streams in our study (e.g. 159-423 m), further supporting previous published ecological data $[55,56]$. Nearly half of total species collected ( $45.7 \%$ or 16 species) were euryzonal or showing wide vertical distributions. These species, for example, S. brevipar, S. whartoni and $S$. bishopi were found occupying more than $80 \%$ (16 streams) of total surveyed streams varying from low to higher altitudes. Simulium tani and S. angulistylum were found at 12 and ten altitudes, respectively (Table 4). A similar trend observed in Thai simuliid species such as $S$. yuphae were found distributed from 650-2534 m [23]. This wide vertical distribution pattern was also observed in other aquatic macroinvertebrates in temperate streams [53]. Black fly species that are widely distributed and adaptable in various physicochemical conditions are likely to be a species complex [57]. This situation has been highlighted in previous studies where the Thai $S$. tani and $S$. angulistylum were found to be a cytological species complexes $[58,59]$. Our results revealed that some of the widespread species (i.e. S. tani, S. angulistylum, S. bishopi) along this altitudinal gradient were also commonly found in other locations in Peninsular Malaysia [31]. Cryptic diversity might be found in Malaysian samples and further cytogenetic and molecular studies would help to clarify this hypothesis.

The observed spatial distributions of preimaginal black flies in our study were predictable on the basis of streamsite characters. Our results revealed that distributions of four common species were related to altitude, temperature, stream size, velocity, streambed particle and discharge. Most of these factors are consistent with the patterns observed in tropical streams in the Oriental region and other geographical regions $[21,25,30]$. Temperature is a wellknown variable that reversely correlated with altitude and has been widely associated with black fly distribution [21, 23, 25, 30, 32]. Moreover, Henriques-Oliveira \& Nessimian [3] indicated that both temperature and stream size were the influencing factors in other aquatic insect distribution and composition in Southeastern Brazil. Based on our observation, species such as Simulium sp. (nr. feuerborni) was largely found in cooler streams (E11-E18) with temperature ranging between $14{ }^{\circ} \mathrm{C}$ and $19{ }^{\circ} \mathrm{C}$ and the mean between $17^{\circ} \mathrm{C}$ and $19{ }^{\circ} \mathrm{C}$. In a broader context, this species was reported as a species complex, which comprised cytoforms A and B in Thailand [60], cytoform $C$ in Malaysia and cytoform D in Indonesia [61]. In particular, 
high frequency of B chromosome was detected in the $S$. feuerborni cytoform $\mathrm{C}$, a unique character for temperate and arctic species [61]. Similarly, S. caudisclerum, S. hackeri, S. digrammicum and S. tanahrataense were inhabitants of cooler streams $[55,62]$. The populations of these high-altitude specialists are fragmented and isolated at high altitude probably as a result of a glacial period, and thus, considered more vulnerable to extinction [8,63]. In contrast, species such as $S$. cheongi was a common inhabitant at normal stream temperatures $\left(23.9-25{ }^{\circ} \mathrm{C}\right)$ [55]. Based on our observation, the availability of larger streams with higher discharge rates gradually decreases with increasing altitude. Species such as $S$. jeffreyi and S. nobile, were largely associated with these stream characters and abundantly found at low altitude. In fact, stream velocity has been emphasized as one of the important factors determining the distribution of black fly larvae [21, 25, 30, 64-66].

Species richness and composition are strongly interconnected with their habitat characteristics [30]. Species richness could increase with altitude but decline at above $1500 \mathrm{~m}$ [2, 6, 7, 23, 67]. Our study corroborated these trends, where species richness started to decline at $1405 \mathrm{~m}$. Similar results found with other macroinvertebrates where taxa richness starts to decrease as altitude increases [3, 68-70]. Regarding species composition observed in cluster analysis, there was a marked separation of streams at similar altitude $(1405 \mathrm{~m})$. This reflects the remarkable change of abiotic factors particularly in the observed water temperature and thus creates a boundary for most of the species with narrow ecological tolerance except several generalists and highly specialised taxa or high-altitude specialists. A similar pattern was reported in other macroinvertebrate studies along altitudinal gradients [70, 71].

Regards to species co-occurrence pattern, the assemblages observed in this study were not random and most of our samplings recorded the co-existence of species (76.6\% or 331), implying high stream environmental heterogeneity (e.g. larger stream with larger streambed particles) [25]. However, species co-existence could also be found in homogenous streams with respect to the availability of microhabitats [40]. A group of species in this particular stream would require similar microhabitat preference $[40,72]$. A recent study indicated that habitat filtering is a major factor that shaping community structure of black flies in tropical streams [66]. Co-existence species usually show similar morphological traits that associate with stream conditions. Our results revealed that most of the co-occurring species possess similar labral fan morphologies, the food-filtering organ that is strongly associated with stream conditions [73]. These species for example, $S$. angulistylum and $S$. trangense were found co-existing in $50 \%$ of total samplings, while other species such as S. tani, S. jeffreyi and Simulium sp (nr. parahiyangum) were found co-occurring in $46 \%$ of total samplings. Therefore, patterns of species assemblage of the black flies in tropical streams in Malaysia mirror previous findings in Thailand and suggest that ecological conditions of the larval habitat play a significant role in determining black fly species assemblage.

\section{Conclusions}

In conclusion, this comprehensive surveillance on the vertical distribution of black flies was conducted for the first time in Peninsular Malaysia and yielded 35 species, representing $42.7 \%$ of total simuliids in Malaysia. The current study has provided new insight into the distribution patterns of preimaginal black fly along an altitudinal gradient in Peninsular Malaysia. Our results indicated that physicochemical characteristics of the stream habitats that are associated with black fly distribution (e.g. stream size, velocity and temperature) varied along an altitudinal gradient. Thus, species diversity and assemblages varied accordingly. We found that certain black fly species are habitat specialists, whereas some are habitat generalists and distributed in wide range of ecological conditions. These species are likely to contain cryptic taxa and further taxonomic study using cytogenetic and molecular methods are required to support this hypothesis. Moreover, this study could deepen our knowledge on the ecology and biology of the specialised taxa in response to environmental changes.

\section{Competing interests \\ The authors declare that they have no competing interests.}

\section{Authors' contributions}

$\mathrm{ZY}, \mathrm{HT}$, and MSA made substantial contribution to conception and design of the work, and collected the specimens. ZY and PP contributed to data analysis and interpretation of data. ZY, VLL, PP, HT and MSA revised the article for important intellectual content. ZY wrote the paper. All authors have read and approved the manuscript.

\section{Acknowledgements}

Thanks are due to Nor Azhar Jamil and Muhammad Rasul Abdullah Halim (Institute Biological Sciences, University of Malaysia) for field assistance. This work was supported by research grants from the University of Malaya (Project No. PG084-2014B and RP003A-13SUS). This project is a part of the first author's PhD research at the University of Malaya, Kuala Lumpur.

\section{Author details}

'Institute of Biological Sciences, Faculty of Science, University of Malaya, 50603 Kuala Lumpur, Malaysia. ${ }^{2}$ Department of Biology, Faculty of Science, Mahasarakham University, Maha Sarakham 44150, Thailand.

Received: 2 February 2016 Accepted: 3 April 2016

Published online: 19 April 2016

\section{References}

1. Hodkinson ID. Terrestrial insects along elevation gradients: species and community responses to altitude. Biol Rev. 2005:80(3):489-513.

2. McCain CM, Grytnes JA. Elevational gradients in species richness. In: Encyclopedia of Life Sciences (ELS). Chichester: John Wiley \& Sons, Ltd; 2010. 
3. Henriques-Oliveira AL, Nessimian JL. Aquatic macroinvertebrate diversity and composition in streams along an altitudinal in Southeastern Brazil. Biota Neotrop. 2010;10(3):115-28.

4. Tomanova S, Tedesco PA, Campero M, Van Damme PA, Moya N, Oberdorff T. Longitudinal and altitudinal changes in macroinvertebrate functional feeding groups in Neotropical streams: a test of the river continuum Concept. Fundamental and applied limnology, arch. Hydrobiol. 2007;170(3):233-41.

5. Barry RG. Mountain climatology and past and potential future climatic changes in mountain regions - a review. Mt Res Dev. 1992;12(1):71-86.

6. Wolda H. Altitude, habitat and tropical insect diversity. Biol J Linn Soc. 1987; 30(4):313-23.

7. McCoy DE. The distribution of insects along elevational gradients. Oikos. 1990;58(3):313-22.

8. Adler PH, Cheke RA, Post RJ. Evolution, Epidemiology, and population genetics of black flies (Diptera: Simuliidae). Infect Gen Evol. 2010;10(7):846-65.

9. Crosskey RW. The natural history of blackflies. Chichester: John Wiley \& Sons Inc; 1990. p. ix+711 pp.

10. Adler PH, Currie DC, Wood DM. The black flies (Simuliidae) of North America. Ithaca: Cornell University Press; 2004.

11. Currie DC, Adler PH. Global diversity of black flies (Diptera: Simuliidae) in freshwater. Hydrobiol. 2008;595(1):469-75.

12. Pramual $P$, Chaliow K, Baimai V, Walton C. Phylogeography of the black fly Simulium tani (Diptera: Simuliidae) from Thailand as inferred from mtDNA sequences. Molecular Ecol. 2005;14(13):3989-4001.

13. Low VL, Adler PH, Takaoka H, Ya'cob Z, Lim PE, et al. Mitochondrial DNA markers reveal high genetic diversity but low genetic differentiation in the black fly Simulium tani Takaoka \& Davies along an elevational gradient in Malaysia. PLoS ONE. 2014;9(6):e100512. doi:10.1371/journal.pone.0100512.

14. Dufrene M, Legendre P. Species assemblages and indicator species: The need for a flexible asymmetrical approach. Ecol Monogr. 1997;67(3):345-66.

15. Hart DD. The adaptive significance of territoriality in filter-feeding larval black flies (Diptera: Simuliidae). Oikos. 1986;46(1):88-92.

16. Colbo MH, Porter GN. Effects of the food supply on the life history of Simuliidae (Diptera). Can J Zool. 1979;57(2):301-6.

17. Ciborowski JJ, Adler PH. Ecological segregation of larval black flies (Diptera: Simuliidae) in northern Saskatchewan, Canada. Can J Zool. 1990;68(10): 2113-22.

18. Halgos J, Illésová D, Krno I. The effect of some ecological factors on longitudinal patterns of black fly community structure (Diptera: Simuliidae) in a foothill stream. Biologia. 2001;56(5):513-23.

19. McCreadie JW, Colbo MH. Larval and pupal microhabitat selection by Simulium truncatum Lundström, S. rostratum Lundström and S. verecundum AA (Diptera: Simuliidae). Can J Zool. 1993;71(2):358-67.

20. Figueiró R, Nascimento ES, Gil-Azevedo LH, Maia-Herzog M, Monteiro RF. Local distribution of blackfly (Diptera: Simuliidae) larvae in two adjacent streams: the role of water current velocity in the diversity of blackfly larvae. Rev Bras Entomol. 2008;52(3):452-4.

21. McCreadie JW, Adler PH, Hamada N. Patterns of species richness for blackflies (Diptera: Simuliidae) in the Nearctic and Neotropical regions. Eco Entomol. 2005:30(2):201-9.

22. Nascimento ES, Figueiró R, Becnel JJ, Araújo-Coutinho CJPC. Influence of temperature on microsporidia infections in a natural population of Simulium pertinax Kollar, 1832 (Diptera; Simuliidae). Braz J Biol. 2007;67(3):519-26.

23. Srisuka W, Takaoka H, Otsuka Y, Fukuda M, Thongsahuan S, Taai K, Choochote W, Saeung A. Seasonal biodiversity of black flies (Diptera: Simuliidae) and evaluation of ecological factors influencing species distribution at Doi Pha Hom Pok National Park, Thailand. Act Trop. 2015;149: 212-9. doi:10.1016/j.actatropica.2015.05.024.

24. Tate CM, Heiny JS. The ordination of benthic invertebrate communities in the South Platte River basin in relation to environmental factors. Freshwater Biol. 1995;33(3):439-54

25. Pramual P, Kuvangkadilok C. Agricultural land use and black fly (Diptera: Simuliidae) species richness and species assemblages in tropical streams, Northeastern Thailand. Hydrobiol. 2009;625(1):173-84

26. Couceiro SRM, Hamada N, Sagot LB, Pepinelli M. Black-fly assemblage distribution in streams in disturbed areas in southern Brazil. Act Trop. 2014; 140:26-33. doi:10.1016/j.actatropica.2014.07.018.

27. McCreadie JW, Adler PH. Scale, time, space, and predictability: species distributions of preimaginal black flies (Diptera: Simuliidae). Oecologia. 1998; 114(1):79-92.
28. McCreadie JW, Adler PH. Ecoregions as predictors of lotic assemblages of blackflies (Diptera: Simuliidae). Ecography. 2006;29(4):603-13.

29. Hamada N, McCreadie JW. Environmental factors associated with the distribution of Simulium perflavum (Diptera: Simuliidae) among streams in Brazilian Amazonia. Hydrobiol. 1999;397:71-8.

30. Hamada N, McCreadie JW, Adler PH. Species richness and spatial distribution of blackflies (Diptera: Simuliidae) in streams of Central Amazonian, Brazil. Fresh Biol. 2002;47(1):31-40.

31. Ya'cob Z, Takaoka H, Pramual P, Low VL, Sofian-Azirun M. Breeding habitat preference of black flies (Diptera: Simuliidae) in Peninsular Malaysia. Act Trop. 2016;153:57-63. doi:10.1016/j.actatropica.2015.10.007.

32. Pramual P, Wongpakam K. Seasonal variation of black fly (Diptera: Simuliidae) species diversity and community structure in tropical streams of Thailand. Entomol Sci. 2010;13(1):17-28.

33. McCreadie JW, Colbo MH. Spatial distribution patterns of larval cytotypes of the Simulium venustum/verecundum complex (Diptera: Simuliidae) on the Avalon Peninsula, Newfoundland: factors associated with occurrence. Can J Zool. 1991;69(10):2651-9.

34. Takaoka H. The Black flies (Diptera: Simuliidae) of Sulawesi, Maluku and Irian Jaya. Fukuoka: Kyushu University Press; 2003. p. xxii + 581 pp.

35. McCreadie JW, Adler PH, Grillet ME, Hamada N. Sampling statistics in understanding distributions of black fly larvae (Diptera: Simuliidae). Act Entomol Serb. 2006;11(Suppl):89-96.

36. Brühl CA, Gunsalam G, Linsenmair KE. Stratification of ants (Hymenoptera, Formicidae) in a primary rain forest in Sabah, Borneo. J Trop Eco. 1998;14(3): 285-97.

37. Brühl CA. Leaf litter ant communities in tropical lowland rain forests in Sabah, Malaysia: Effects of forest disturbance and fragmentation. PhD Thesis. Würzburg: Julius-Maximilians-Universität; 2001.

38. Gotelli NJ, Entsminger GL. EcoSim: null models software for ecology, v.7. Acquired Intelligence Inc and Kesey-Bear [software on the Internet]. Published by the authors. [cited 2015 Sept 20]. Available from: garyentsminger.com/Ecosim; 2009.

39. Stone $L$, Roberts $A$. The checkerboard score and species distributions. Oecologia. 1990;85(1):74-9.

40. Figueiró R, Gil-Azevedo LH, Maia-Herzog M, Monteiro RF. Diversity and microdistribution of black fly (Diptera: Simuliidae) assemblages in the tropical savanna streams of the Brazilian Cerrado. Mem Inst Oswaldo Cruz. 2012;107(3):362-9.

41. McCreadie JW, Hamada N, Grillet ME. Spatial-temporal distribution of preimaginal blackflies in Neotropical streams. Hydrobiol. 2004;513(1): 183-96.

42. McCune B, Mefford MJ. PC-ORD. Multivariate Analysis of Ecological Data, Version 5.14. Gleneden Beach: MjM Software; 2006.

43. Seaby RM, Henderson PA. Species Diversity and Richness, Ver. 4. 2006.

44. Adler PH, Crosskey RW. World blackflies (Diptera: Simuliidae): A comprehensive revision of the taxonomic and geographical inventory [2015]. 120 pp. Available from: http://www.clemson.edu/cafls/biomia/pdfs/ blackflyinventory.pdf (accessed on $1^{\text {st }}$ July 2015).

45. Fukuda M, Choochote W, Bain O, Aoki C, Takaoka H. Natural infections with filarial larvae in two species of black flies (Diptera: Simuliidae) in northern Thailand. Jpn J Trop Med Hyg. 2003;31(2):99-102.

46. McCreadie JW, Adler PH. Spatial distribution of rare species in lotic habitats. Insect Conserv Divers. 2008;1(3):127-34.

47. Gotelli NJ, Simberloff D. The distribution and abundance of tallgrass prairie plants: a test of the core- satellite hypothesis. Am Nat. 1987;130(1):18-35.

48. Gaston KJ, Lawton JH. Patterns in the distribution and abundance of insect populations. Nature. 1988;331(6158):709-12

49. Hutchinson GW. Concluding remarks. Cold Spring Harb Symp Quant Biol. 1957:22:415-27.

50. Hubbell SP. Neutral theory in community ecology and the hypothesis of functional equivalence. Funct Ecol. 2005;19(1):166-72.

51. Grillet ME, Barrera R. Spatial and temporal abundance, substrate partitioning and species co-occurrence in a guild of Neotropical blackflies (Diptera: Simuliidae). Hydrobiol. 1997;345(2):197-208.

52. Scheder C, Waringer JA. Distribution patterns and habitat characterization of Simuliidae (Insecta: Diptera) in a low-order sandstone stream (Weidlingbach, Lower Austria). Limnologica. 2002;32(3):236-47.

53. Dudgeon D. Tropical Stream Ecology. London: Academic; 2008. p. 316.

54. Low VL, Takaoka H, Adler PH, Ya'cob Z, Norma-Rashid Y, Chen CD, SofianAzirun M. A multi-locus approach resolves the phylogenetic relationships of 
the Simulium asakoae and Simulium ceylonicum species groups in Malaysia: evidence for distinct evolutionary lineages. Med Vet Entomol. 2015;29(3): 330-7.

55. Takaoka H, Davies DM. The Black Flies (Diptera: Simuliidae) of West Malaysia. Fukuoka: Kyushu University press; 1995. p. viii + 175pp.

56. Jitklang S, Kuvangkadilok C. A new species of Simulium (Gomphostilbia) (Diptera: Simuliidae) from southern Thailand, with description of its polytene chromosomes. Stud Dipterol. 2007;14(2):369-75.

57. Adler PH, McCreadie JW. The hidden ecology of black flies: Sibling species and ecological scale. Am Entomol. 1997;43(3):153-62.

58. Tangkawanit U, Kuvangkadilok C, Baimai V, Adler PH. Cytosystematics of the Simulium tuberosum group (Diptera; Simuliidae) in Thailand. Zool J Linn Soc. 2009;155(2):289-315.

59. Pramual P, Kuvangkadilok C. Integrated cytogenetic, ecological, and DNA barcode study reveals cryptic diversity in Simulium (Gomphostilbia) angulistylum (Diptera: Simuliidae). Genome. 2012;55(6):447-58.

60. Pramual P, Wongpakam K. Population genetic of the high elevation black fly Simulium (Nevermannia) feuerborni Edwards in Thailand. Entomol Sci. 2013;16(3):298-308.

61. Pramual P, Thaijaren J, Sofian-Azirun M, Ya'cob Z, Hadi UK, Takaoka H. Cytogenetic and molecular evidence of additional cryptic diversity in high elevation black fly Simulium feuerborni (Diptera: Simuliidae) populations in Southeast Asia. J Med Entomol. 2015;52(5):829-36.

62. Takaoka H, Sofian-Azirun M, Ya'cob Z, Hashim R. Two new species of Simulium (Gomphostilbia) (Diptera: Simuliidae) from Cameron's Highlands, Peninsular Malaysia, with keys to 21 species of the Simulium asakoae species-group. Zootaxa. 2014;3765(1):054-68.

63. Finn DS, Adler PH. Population genetic structure of a rare high-elevation black fly, Metacnephia coloradensis, occupying Colorado lake outlet streams. Freshwater Biol. 2006;51(12):2240-51.

64. Zhang Y, Malmqvist B, Englund G. Ecological process affecting community structure of blackfly larvae in regulated and unregulated rivers: a regional study. J App Ecol. 1998;35(5):673-86.

65. Palmer RW, Craig DA. An ecological classification of primary labral fans of filter-feeding black fly (Diptera: Simuliidae) larvae. Can J Zool. 2000;78(2): 199-218.

66. Pangjanda S, Pramual P. Trait-based and phylogenetic community ecology of black flies (Diptera: Simuliidae) in Tropical streams of Thailand. Hydrobiol. 2015;763(1):345-56.

67. Lawton JH, MacGarvin M, Heads PA. Effects of altitude on the abundance and species richness of insect herbivores on bracken. J Anim Ecol. 1987; 56(1):147-60.

68. Jacobsen D, Schultz R, Encalada A. Structure and diversity of stream invertebrate assemblages: the influence of temperature with altitude and latitude. Freshwater Biol. 1997;38(2):247-61.

69. Jacobsen D. Contrasting patterns in local and zonal family richness of streams invertebrates along an Andean altitudinal gradient. Freshwater Biology. 2004;49(10):1293-305.

70. Huamantinco AA, Nessimian JL. New Neotropical genus and species of Odontocerinae (Trichoptera: Odontocerinae) from Southeastern Brazil. Aquatic Insect. 2004;26(3/4):281-8.

71. Palmer C, Palmer A, O'Keeffe J, Palmer R. Macroinvertebrate community structure and altitudinal changes in the upper reach of warm temperate southern African river. Freshwater Biol. 1994;32(2):337-47.

72. Pramual P, Kuvangkadilok C, Jitklang S, Tangkawanit U, Adler PH. Geographical versus ecological isolation of closely related black flies (Diptera: Simuliidae) inferred from phylogeny, geography, and ecology. Org Divers Evol. 2012;12(2):183-95.

73. Zhang Y, Malmqvist B. Relationships between labral fan morphology and habitat in North Swedish blackfly larvae (Diptera: Simuliidae). Biol J Linn Soc. 1996:59(3):261-80

\section{Submit your next manuscript to BioMed Central and we will help you at every step:}

- We accept pre-submission inquiries

- Our selector tool helps you to find the most relevant journal

- We provide round the clock customer support

- Convenient online submission

- Thorough peer review

- Inclusion in PubMed and all major indexing services

- Maximum visibility for your research

Submit your manuscript at www.biomedcentral.com/submit
Biomed Central 